Pure and Applied Mathematics Quarterly

Volume 6, Number 3

(Special Issue: In honor of

Joseph J. Kohn, Part 1 of 2$)$

$815-827,2010$

\title{
The Spectrum and Pseudospectrum of Non-self Adjoint Pseudodifferential Operators
}

\author{
Charles L. Epstein \\ Dedicated to Professor Joe Kohn on the occasion of his 75th birthday
}

\begin{abstract}
We study the spectrum of relatively compact, non-self adjoint perturbations of self adjoint operators, with the formal properties of pseudodifferential operators. When the self adjoint operator is elliptic, we obtain an estimate for the location of the spectrum in complex plane, which provides a quantitative improvement of classical results of Keldyš, Gohberg and Krein.
\end{abstract} Keywords: spectrum, non-self adjoint operator, pseudodifferential operator, resolvent kernel

\section{INTRODUCTION}

If $A: X \rightarrow Y$ is a closed operator from a dense domain $D(A) \subset X$, to another Banach space $Y$, then the resolvent set, the complement of the spectrum, is the set of points $\lambda \in \mathbb{C}$, such that $A-\lambda$ has a bounded inverse, $R_{A}(\lambda)=(A-\lambda)^{-1}$. The operator $R_{A}(\lambda)$ is called the resolvent of $A$. We denote the resolvent set by $\rho(A)$ and its complement, the spectrum of $A$, by $\Lambda(A)$. If $X=Y$ is a Hilbert space, with inner product $\langle\cdot, \cdot\rangle$, then we say that $A$ is self adjoint if

$$
\langle A x, y\rangle=\langle x, A y\rangle \text { for all } x, y \in D(A),
$$

and the linear functional $\ell(x)=\langle A x, y\rangle$ is bounded only if $y \in D(A)$. In this case it is easy to see that the spectrum lies in $\mathbb{R}$. The norm of the resolvent operator

Received January 30, 2008

Research partially supported by NSF grant DMS06-03973 and by DARPA HR00110510057. 
of a self adjoint operator satisfies a precise bound:

$$
\left\|R_{A}(\lambda)\right\|=\frac{1}{\operatorname{dist}(\lambda, \Lambda(A))} .
$$

If the operator is not self adjoint, then no such estimate holds.

In recent years there has been a resurgence of interest in the spectral theory of non-self adjoint operators. Much of this interest stems from the desire to numerically compute the spectra of such operators, or their finite dimensional approximations. In a finite dimensional context, doing a computation with a relative machine accuracy of $\epsilon$, one cannot distinguish the spectrum of $A$ :

$$
\{\lambda \in \mathbb{C}: \text { there exists } v \neq 0 \text { such that }(A-\lambda) v=0\}
$$

from the set

$$
\{\lambda \in \mathbb{C}: \text { there exists } v \neq 0 \text { such that }\|(A-\lambda) v\| \leq \epsilon\|v\|\}
$$

This explains, in part, why the spectrum itself may not be as useful a concept as the pseudospectrum, which we now define: For an $\epsilon>0$ we define the $\epsilon$ pseudospectrum of $A$ to be

(5) $\Lambda_{\epsilon}(A)=\{\lambda \in \mathbb{C}:$ there is a $v \neq 0 \in D(A)$ such that $\|(A-\lambda) v\| \leq \epsilon\|v\|\}$,

or, equivalently

$$
\lambda \in \Lambda_{\epsilon}(A) \text { if } \lambda \in \Lambda(A) \text { or }\left\|R_{A}(\lambda)\right\| \geq \epsilon^{-1} .
$$

Clearly, for every $\epsilon>0, \Lambda(A) \subset \Lambda_{\epsilon}(A)$. If $A$ is self adjoint, then $\Lambda_{\epsilon}(A)$ is just the $\epsilon$-neighborhood of $\Lambda(A)$. From many examples, it is now well understood that, if $A$ is not self adjoint, then $\Lambda_{\epsilon}(A)$ can be much larger than $A$. An example in [7], shows that $\Lambda_{\epsilon}(A)$ could be the convex set, $x \geq y^{2}$, whereas $\Lambda(A)$ is a discrete subset of $[0, \infty)$.

Inspired by the work of Davies, Dencker, Sjöstrand, and Zworksi have given a very precise characterization, in the context of semi-classical pseudodifferential operators, of the semi-classical pseudospectrum in terms of the semi-classical principal symbol, see [1, 6, 2]. The monograph of Gohberg and Krein, [3] is the definitive classical text on non-self adjoint operators. Trefethen and Embree's text, [5], provides a more modern and comprehensive treatment, from an applied perspective. 
In this note we prove a result, describing the location of the spectrum and pseudospectrum for operators modeled on perturbations of elliptic, self adjoint pseudodifferential operators. Our main result is a refinement of classical results of Keldys̆, with extensions due to Gohberg and Krein. In modern language, their result is:

Theorem 1 (Keldys̆, Gohberg and Krein). Let $A=L+T$, where $L$ is an unbounded self-adjoint operator, with dense domain $D(L) \subset H$. We suppose that $L$ is invertible, has purely discrete spectrum, and that $T L^{-1}$ is compact. Moreover, $L^{-1} T L^{-1}$ belongs to the $p$-Schatten class for some $p$. In this case, the resolvent set of $A$ is non-empty, and the entire spectrum of $A$ consists of generalized eigenvalues of finite multiplicity. For any $\epsilon>0$, all but finitely many points of this spectrum lies in the sectors

$$
F_{\epsilon}=\{\sigma+i \tau:|\tau| \leq \epsilon|\sigma|\}
$$

The span of the generalized eigenvectors is a dense subspace of $H$. If $L$ is a nonnegative, then all, but finitely many, eigenvalues of $A$ lie in $\operatorname{Re} \lambda>0$.

See [3][Theorem V.10.1]. In our analysis we make a slightly stronger hypothesis on the operator $L$, namely that it is, in some sense, an "elliptic" operator.

For example our result applies to an operator of the form $A=L+T$, where $L \in \Psi^{r}(X ; E)$, and $T \in \Psi^{s}(X ; E), 0 \leq s<r$, here $X$ is a compact manifold, $E \rightarrow X$ a vector bundle. The operator $L$ is assumed to be elliptic and self adjoint. In this case we show that, there are constants $C_{1}, C_{2}$ so that:

$$
\Lambda(A) \subset\left\{(\sigma+i \tau):|\tau| \leq\left(C_{1}+C_{2}|\sigma|\right)^{\frac{s}{r}}\right\} .
$$

If $L$ is positive, then we show that the spectrum lies in a right half plane and satisfies this sort of estimate.

We also show that the norm of the resolvent decays in proportion to the distance from a set of the type described in (8), thus, for any positive $\epsilon$, the $\epsilon$ pseudospectrum lies in a similar set. It should be noted that the order of magnitude of the growth of $\operatorname{Im} \lambda$ as a function $\operatorname{Re} \lambda$ in (8) is sharp.

Example 1. If $P_{\alpha}=i \partial_{x}+\pi \alpha$ on the domain $H^{1}\left(S^{1}\right) \subset L^{2}\left(S^{1}\right)$, and $Q_{s}=f_{s}\left(P_{\alpha}\right)$, where $f_{s}(x)=i|x|^{s}$, then $P_{\alpha}+Q_{s}$ satisfies the hypotheses of our theorem and

$$
\Lambda\left(P_{\alpha}+Q_{s}\right)=\left\{(2 k+\alpha) \pi+i|(2 k+\alpha) \pi|^{s}: k \in \mathbb{Z}\right\} .
$$


As $\alpha$ varies in $(0,2)$, the spectra of $\Lambda\left(P_{\alpha}+Q_{s}\right)$ trace the boundary of the set $|\tau|=|\sigma|^{s}$. As $P_{\alpha}+Q_{s}$ are normal operators, their resolvents also satisfy (2). Replacing $f_{s}$ with $f_{s, \beta}=e^{i \pi \beta x}|x|^{s}$, any point within the set $|\tau| \leq|\sigma|^{s}$ appears, for some $\alpha, \beta \in(0,2)$, in the spectrum of $P_{\alpha}+f_{s, \beta}\left(P_{\alpha}\right)$.

The main technical tool we use is an interpolation inequality, and so we state our results in a fairly general, functional analytic setting. In the next section we cover the functional analytic preliminaries; in the last section we state and prove our main theorems, and give several examples.

Remark 1. In what follows we use $C$ to denote a variety of positive constants, which do not depend on the point of evaluation, i.e. $x$.

\section{Functional Analytic Preliminaries}

We let $\left(H_{0},\|\cdot\|_{0}\right)$ denote a separable Hilbert space, and $M$ a positive, unbounded, self adjoint operator with domain $D(M) \subset H_{0}$. Suppose that

(1) $M$ is invertible, hence there is a constant $C$ so that $\|M x\|_{0} \geq C\|x\|_{0}$, for all $x \in D(M)$,

(2) $M$ has a compact resolvent,

(3) Some power of $M^{-1}$ is a trace class operator.

We then call $M$ an admissible operator. Let $H_{1}=D(M)$ with the norm defined by $\|x\|_{1}=\|M x\|_{0}$. We define the Hilbert spaces $\left\{H_{0} \supset H_{s} \supset H_{1}: s \in(0,1)\right\}$, by complex interpolation. From the definition of the norm, it follows easily that $H_{s}=D\left(M^{s}\right)$, and we have the estimate

$$
\|x\|_{s} \leq\left\|M^{s} x\right\|_{0}
$$

Indeed, by the closed graph theorem, the norm on $H_{s}$ is equivalent to $\left\|M^{s} x\right\|_{0}$. We call the Hilbert spaces $\left\{H_{s}: s \in[0,1]\right\}$, with norm given by $\left\|M^{s} x\right\|_{0}$, the scale of spaces defined by the (admissible) operator $M$. As $M$ is self adjoint and $M^{-1}$ is compact, it is clear that, for $0<s<1$, the operators $M^{-s}$ are also compact. This shows that the unit ball in $H_{s}, s>0$, is a compact subset of $H_{0}$.

A standard application of the Phragmen-Lindelöf Theorem proves the following interpolation estimate 
Proposition 1. Let $0<s<t \leq 1$ and suppose that $x \in H_{t}$, then

$$
\|x\|_{s} \leq\|x\|_{0}^{1-\frac{s}{t}}\|x\|_{t}^{\frac{s}{t}}
$$

We apply the generalized Peter-Paul inequality to conclude

Corollary 1. If $0 \leq s<t \leq 1$, then there is a constant $C_{s, t}$, such that, for any $\epsilon>0, x \in H_{t}$, we have

$$
\|x\|_{s} \leq C_{s, t}\left[\epsilon^{\frac{s}{s-t}}\|x\|_{0}+\epsilon\|x\|_{t}\right] .
$$

Definition 1. A closed operator $B$ with domain $D(B) \subset H_{0}$, is of order (at most) $s \leq 1$ if $D(B) \supset H_{s}$, and, for $u \in[s, 1]$ there are constants $C_{u}$ so that

$$
\|B x\|_{u-s} \leq C\|x\|_{u} \text { for all } x \in H_{u} .
$$

Definition 2. A closed operator $B$ is elliptic of order $s \leq 1$, if $D(B) \subset H_{s}$, and there is a constant $C$ so that

$$
\|x\|_{s} \leq C\left[\|B x\|_{0}+\|x\|_{0}\right] \text { for all } x \in D(B) .
$$

Remark 2. The norm $\|\cdot\|_{s}$ is defined by the operator $M_{s}$, so our definition of the order of an elliptic operator only agrees with the usual definition when $B$ is a classical elliptic pseudodifferential operator, and $M$ is itself a classical elliptic pseudodifferential operator of order 1 . It might be more in keeping with standard practice to call these operators "sub-elliptic" of order $s$. For economy of exposition we use the simpler terminology.

Proposition 2. If $B$ is an elliptic operator of order $0<s \leq 1$, then the unit ball, in the graph of $B$, with respect to the graph norm, is compact in $H_{0}$. If the resolvent set of $B$ is non-empty, then the resolvent of $B$ is a compact operator, and there is a $p>0$ such that for $\lambda \in \rho(B)$, the operator $(B-\lambda)^{-p}$ is trace class.

Proof. The facts that the unit ball in $H_{s}$ is compact in $H_{0}, D(B) \subset H_{s}$, and the estimate (14) show that the unit ball in $D(B)$, with respect to the graph norm, is compact in $H_{0}$. If the resolvent set of $B$ is non-empty, then $B$ has a compact resolvent.

If $\rho(B) \neq \emptyset$, then choose $\lambda \in \rho(B) \cap \mathbb{R}$, and let $\left\{b_{j}^{2}\right\}$ denote the eigenvalues of the non-negative, self adjoint operator $\left(B^{*}-\lambda\right)(B-\lambda)$, and $\left\{m_{j}\right\}$ the eigenvalues 
of $M$, both in increasing order. The triangle inequality implies that

$$
\begin{aligned}
\langle(B-\lambda) x,(B-\lambda) x\rangle & \geq\|B x\|_{0}^{2}-\lambda^{2}\|x\|_{0}^{2} \\
& \geq \frac{1}{2 C^{2}}\left\|M^{s} x\right\|_{0}^{2}-\left[\lambda^{2}+C^{-2}\right]\|x\|_{0}^{2} .
\end{aligned}
$$

The second line follows from the ellipticity assumption, the definition of $\|\cdot\|_{s}$, and the arithmetic-geometric mean inequality. As $D(B) \subset H_{s}$, the min-max characterization of eigenvalues shows that

$$
b_{j}^{2} \geq \max \left\{\frac{m_{j}^{2 s}}{2 C^{2}}-\left(\lambda^{2}+C^{-2}\right), 0\right\} .
$$

For some $p>1$ the sum

$$
\sum_{j=1}^{\infty} m_{j}^{-p}
$$

converges. This fact and (16) imply that

$$
\sum_{j=1}^{\infty} b_{j}^{-\frac{p}{s}}<\infty
$$

Hence, $(B-\lambda)$ belongs to a $q$-Schatten class, for sufficiently large $q$.

In the sequel we study operators of the form $A=L+T$. Here $L$ is a self adjoint, elliptic operator of order $t$ and $T$ is a closed operator of order $s<t$. The definitions of order and ellipticity imply that

$$
D(B) \supset H_{s} \supset H_{t} \supset D(L) .
$$

We do not assume that $L$ is non-negative. Indeed, $L$ given by a classically elliptic, or sub-elliptic first order system is a important example of the sort of operator we are considering. The operator $T(L-i)^{-1}$ is compact and therefore $(L-i)^{-1} T(L-$ $i)^{-1}$ belongs to the same $p$-Schatten class as $(L-i)^{-1}$. It follows from classical results that $A$, with $D(A)=D(L)$, is a closed operator. Theorem 1 shows that $A$ has a compact resolvent, such that for any $\epsilon>0$, there is an $r_{\epsilon}>0$ such that the spectrum of $A$ lies in $F_{\epsilon} \cup B_{r_{\epsilon}}(0)$. 


\section{Estimates on the SPECTRUm AND RESOlVENT KeRNEL}

With the preliminaries above we state and prove our main results. Let $(M, D(M))$, with $D(M) \subset H_{0}$, be an admissible operator, and $\left\{H_{s}\right\}$ the scale of Hilbert spaces defined by $M$. In the sequel the notation $\|x\|$ is used for $\|x\|_{0}$.

Theorem 2. Let $(L, D(L))$, be a self adjoint elliptic operator of order $0<t \leq$ 1 and $(T, D(T))$ a closed operator of order $s<t$. The operator $A=L+T$, with $D(A)=D(L)$ is a closed operator with a compact resolvent. The spectrum of $A$ consists of generalized eigenvalues with finite multiplicity, the span of the generalized eigenspaces is dense in $H_{0}$. There are constants $C_{1}, C_{2}$ so that

$$
\Lambda(A) \subset\left\{\sigma+i \tau:|\tau| \leq C_{1}\left(|\sigma|+C_{2}\right)^{\frac{s}{t}}\right\}
$$

If $L$ is non-negative, then all but finitely many eigenvalues lies in $\operatorname{Re} \lambda>0$, and we can replace $|\sigma|$, with $\sigma$ on the right hand side of (19).

Using a similar argument, we can also estimate $R_{A}(\lambda)=(A-\lambda)^{-1}$. This theorem shows that the pseudospectra of $A$ lie in the some sort of sets.

Theorem 3. Under the hypotheses of Theorem 2, there are constants $C_{1}, C_{2}$ so that for $\sigma+i \tau \in \rho(A)$,

$$
\left\|R_{A}(\sigma+i \tau)\right\| \leq \frac{C_{2}}{\left.\sqrt{\tau^{2}-C_{1}\left(1+|\sigma|^{\frac{2 s}{t}}\right.}\right)} .
$$

We now give the proofs of these results

Proof of Theorem 2. As $T$ is of order $s<t$, we apply Corollary 1 to conclude that for any $\epsilon>0$ there is a $C_{\epsilon}$ such that, for any $x \in D(L) \subset H_{t}$, we have the estimate

$$
\begin{aligned}
\|T x\|_{0} & \leq\|x\|_{s} \\
& \leq \epsilon\|x\|_{t}+C_{\epsilon}\|x\|_{0} \\
& \leq C \epsilon\|L x\|_{0}+C_{\epsilon}^{\prime}\|x\|_{0} .
\end{aligned}
$$

In the last line we use the ellipticity of $L$. This shows that $T$ is $L$-bounded, with relative bound $\epsilon$, for any $\epsilon>0$. Applying Theorem IV.1.1 from [4] we conclude that $(A, D(L))$ is a closed operator. 
Replacing $L$ by $L-\alpha$, for a real number $\alpha$, we can assume that $L$ is invertible. For $L$ invertible, the ellipticity easily implies that there is a constant $C$ so that

$$
\|x\|_{t} \leq C\|L x\| \text { for } x \in D(L),
$$

which is equivalent to

$$
\left\|L^{-1} y\right\|_{t} \leq C\|y\| \text { for } y \in H_{0} .
$$

To see that the operator $T L^{-1}$ is compact, we observe that, as $T$ is order $s$, and $D(L) \subset H_{t}$, we can apply (12) and (23) to conclude that, for some constant $C$,

$$
\begin{aligned}
\left\|T L^{-1} y\right\|_{t-s} & \leq C\left\|L^{-1} y\right\|_{t} \\
& \leq C\|y\| .
\end{aligned}
$$

Hence $T L^{-1}: H_{0} \rightarrow H_{t-s}$ boundedly. As the inclusion $H_{t-s} \rightarrow H_{0}$ is compact, $T L^{-1}: H_{0} \rightarrow H_{0}$ is as well. Finally, as $L^{-1}$ belongs to the $p$-Schatten class we conclude that $L^{-1} T L^{-1}$ does as well. Hence we can apply Theorem 1 to obtain all the stated results but the estimate for the location of the spectrum.

As the spectrum of $A$ consists of generalized eigenvalues of finite multiplicity, if $\lambda=\sigma+i \tau \in \Lambda(A)$, then there exists a unit vector $x \in D(L) \subset H_{t}$ such that $(A-\lambda) x=0$. Because $L$ is self adjoint, $\langle x, L x\rangle$ is real; this implies two identities:

$$
\langle L x, L x\rangle+\operatorname{Re}\langle T x, L x\rangle=\sigma\langle x, L x\rangle
$$

and

$$
\operatorname{Im}\langle T x, x\rangle=\tau\langle x, x\rangle
$$

Equation (25) and the fact that $\|x\|=1$ imply that

$$
|\sigma|\|L x\| \geq\|L x\|^{2}-\|T x\|\|L x\| .
$$

Assuming that $L x \neq 0$, we see that this estimate and the fact that $T$ is an operator of order $s$ imply that

$$
|\sigma| \geq\|L x\|-C\|x\|_{s} .
$$

Using Corollary 1, and the ellipticity of $L$ it follows from (28) that for any $\epsilon>0$, there is a constant $C_{\epsilon}$ so that

$$
|\sigma| \geq(1-\epsilon)\|L x\|-C_{\epsilon}\|x\|
$$


Finally, as $L$ is elliptic of order $t$ this implies that there are constants $C_{1}, C_{2}$ so that

$$
\|x\|_{t} \leq C_{1}|\sigma|+C_{2}\|x\| .
$$

As $L x=0$ can only be true for $x$ belonging to a finite dimensional subspace, (30) holds in this case as well.

To complete the proof, we observe that (26), the Cauchy-Schwarz inequality and the fact that $T$ is of order $s$ imply that

$$
|\tau| \leq C\|x\|_{s} .
$$

The interpolation inequality, (11), (30), and the fact that $x \in H_{t}$ imply that

$$
\begin{aligned}
|\tau| & \leq C\|x\|_{t}^{\frac{s}{t}} \\
& \leq C\left[C_{1}|\sigma|+C_{2}\right]^{\frac{s}{t}} .
\end{aligned}
$$

This completes the proof, but for the remarks about the case $L \geq 0$. If $L$ is non-negative, then Theorem 1 shows that only finitely many points in $\Lambda(A)$ can lie in $\operatorname{Re} \lambda<0$, so this case follows as well.

Now we turn to the proof of Theorem 3 .

Proof of Theorem 3. The assumption $L$ is elliptic of order $t>s$ implies that there is a constant, $C$, so that, for $x \in D(L)$ we have the estimate

$$
\|x\|_{t} \leq C[\|L x\|+\|x\|] .
$$

As $L$ is self adjoint with compact resolvent, replacing $L$ by $L-\lambda_{0}$, where $\lambda_{0} \in$ $\mathbb{R} \cap \Lambda(L)^{c}$ we obtain the estimate in (33) without $\|x\|$ on the right hand side:

$$
\|x\|_{t} \leq C\|L x\| .
$$

Shifting $L$ in this manner does not affect the conclusion of the theorem, so we assume that the stronger inequality, (34) holds.

We need a lower bound on $\|(A-(\sigma+i \tau)) x\|$. The Cauchy-Schwarz inequality and the Peter-Paul inequality imply that, for any $\epsilon>0$, and $x \in D(A)=D(L)$

$$
\|(L+T-(\sigma+i \tau)) x\|^{2} \geq(1-\epsilon)\|(L-(\sigma+i \tau)) x\|^{2}+\left(1-\frac{1}{\epsilon}\right)\|T x\|^{2} .
$$

As $L$ is self adjoint we have the identity

$$
\|(L-(\sigma+i \tau)) x\|^{2}=\|(L-\sigma) x\|^{2}+\tau^{2}\|x\|^{2} .
$$


That fact that $T$ is of order $s$, the ellipticity of $L$, and the interpolation inequality imply that

$$
\|T x\|^{2} \leq C\|x\|^{2\left(1-\frac{s}{t}\right)}\|L x\|^{\frac{2 s}{t}} .
$$

Writing $L x=(L-\sigma) x+\sigma x$ we can apply the triangle inequality to conclude that

$$
\|T x\|^{2} \leq C\|x\|^{2\left(1-\frac{s}{t}\right)}\left[\|(L-\sigma) x\|^{\frac{2 s}{t}}+(|\sigma|\|x\|)^{\frac{2 s}{t}}\right] .
$$

Employing the generalized Peter-Paul inequality, this estimate implies that there is a constant $C_{\epsilon}$ so that

$$
\left(\frac{1}{\epsilon}-1\right)\|T x\|^{2} \leq(1-\epsilon)\|(L-\sigma) x\|^{2}+C_{\epsilon}\left(1+|\sigma|^{\frac{2 s}{t}}\right)\|x\|^{2} .
$$

Combining this estimate with (35) and (36) we see that

$$
\|(L+T-(\sigma+i \tau)) x\|^{2} \geq\left[(1-\epsilon)|\tau|^{2}-C_{\epsilon}\left(1+|\sigma|^{\frac{2 s}{t}}\right)\right]\|x\|^{2} .
$$

This estimate shows that there are constants $C_{1}, C_{2}$ so that, for $(\sigma+i \tau) \in \rho(A)$, we have the bound

$$
\left\|(A-(\sigma+i \tau))^{-1}\right\| \leq \frac{C_{1}}{\left.\sqrt{|\tau|^{2}-C_{2}\left(1+|\sigma|^{\frac{2 s}{t}}\right.}\right)},
$$

which completes the proof of the theorem.

From the definition of pseudospectrum, the following corollary is an immediate consequence of Theorem 3 :

Corollary 2. Under the hypotheses of Theorem 3, if $\epsilon>0$, and $(\sigma+i \tau) \in \Lambda_{\epsilon}(A)$, then

$$
\tau^{2} \leq C_{2}|\sigma|^{\frac{2 s}{t}}+\epsilon^{2} C_{1}^{2}+C_{2} .
$$

Remark 3 . The estimate on the pseudospectrum is of course not very sharp, given that the spectrum is a discrete set, but is meant to illustrate that spectrum and pseudospectrum are subject to the same constraints.

Remark 4. There is a standard estimate showing that the resolvent is bounded by the reciprocal of the distance to the numerical range

$$
\Theta_{A}=\{\langle A x, x\rangle: x \in D(A) \text { with }\|x\|=1\} .
$$

It is a classical result of Hausdorff that $\Theta_{A}$ is a convex subset of $\mathbb{C}$. If $L$ is nonnegative, then the numerical range of $A$ is often a proper convex cone. The 
estimate in (41) is then a refinement of the classical result. However if $L$ is indefinite, with spectrum tending to $\pm \infty$, then it is often the case that $\Theta_{A}=\mathbb{C}$, and the classical result offers no estimate on the norm of the resolvent. In this case, Theorem 3 is more than a small improvement on a classical result.

We close with a few concrete examples.

Example 2. If $T$ is an operator of order 0, that is a bounded operator, then we see that the spectrum of $L+T$ lies in a bounded neighborhood of the real axis. This follows easily from the estimate (31).

Example 3. Let $H_{0}=L^{2}(\mathbb{R})$ and $L=-\partial_{x}^{2}+x^{2}, H_{1}=D(L)$ (the maximal domain of $L$ ). If we let

$$
T=a(x)\left(\partial_{x}-x\right)+b(x)\left(\partial_{x}+x\right),
$$

where $a, b$ are bounded smooth functions on $\mathbb{R}$, then clearly $T$ is an operator of order $\frac{1}{2}$. As $L$ is a positive operator, it follows from Theorem 2 that, for some constants $C_{1}, C_{2}$, the spectrum of $A=L+T$ lies in a set of the form:

$$
|\tau| \leq C_{1}\left(C_{2}+\sigma\right)^{\frac{1}{2}} \text { where } \sigma>-C_{2} .
$$

More generally, if $L$ is itself an admissible operator, then we can take $M=L$. The theorem applies to any closed operator $T$, such that $D(L) \subset D(T)$, and for some $s<1,0 \leq a \leq 1-s$, there are constants $k_{a}$ so that

$$
\left\|L^{a} T x\right\| \leq k_{a}\left\|L^{s+a} x\right\| \text { for all } x \in D(L) .
$$

Theorem 2 then shows that, for constants $C_{1}, C_{2}$, we have:

$$
\Lambda(L+T) \subset\left\{\sigma+i \tau:|\tau| \leq C_{1}\left(C_{2}+\sigma\right)^{s}\right\} \cap\left\{\sigma+i \tau: \sigma>-C_{2}\right\} .
$$

In this case the coefficient $C_{1}$ can be taken as close as we like to the constant $k_{0}$, appearing in (46).

Example 4. Let $X$ be a compact manifold, $E \rightarrow X$ a vector bundle. We let $H^{s}(X ; E)$ denote the classical $L^{2}$-Sobolev spaces on sections of $E$. These spaces are well known to be complex interpolation spaces. Suppose that $L \in \Psi^{k}(X ; E)$ is a self adjoint, Kohn-Nirenberg pseudodifferential operator with domain $D(L)$. We take $H_{1}=H^{k}(X ; E)$. It suffices for $L$ to be sub-elliptic, that is, for some 
$r \leq k$, we have $D(L) \subset H^{r}(X ; E)$, and there is a constant $C$ so that for every $f \in D(L)$ we have the estimate

$$
\|f\|_{r} \leq C\left[\|L f\|_{0}+\|f\|_{0}\right] .
$$

If $T \in \Psi^{s}(X ; E)$ with $s<r$, then we can apply the results above to conclude that $A=L+T$ with domain $D(L)$ is a closed operator with a compact resolvent. The spectrum and $\epsilon$-pseudospectra of $A$ lie in sets of the form

$$
\left\{\sigma+i \tau:|\tau| \leq\left(C_{1}+C_{2}|\sigma|\right)^{\frac{s}{r}}\right\} .
$$

Example 5. Let $Y$ be a compact Riemannian manifold and $X \subset Y$ a bounded domain with a smooth boundary. Let $L^{\prime}$ be an formally elliptic, non-negative, self adjoint second order operator defined on $\mathcal{C}^{\infty}(Y)$. For $L$ we take a self adjoint extension of $L^{\prime}\left\lceil_{\mathcal{C}_{\mathrm{c}}^{\infty}(X)}\right.$, defined by an elliptic boundary condition. The domain of $L$ is contained in $H^{2}(X)$, and therefore $L$ has a compact resolvent. We let $H_{0}=L^{2}(X)$ and $H_{1}=H^{2}(X)$. The spaces $H_{a}=H^{2 a}(X), a \in[0,1]$ are the interpolation spaces for this pair. This does not quite fit the model described above: the necessity for boundary conditions prevents the norms on the Sobolev spaces $H^{2 a}(X)$ from being defined by the powers of fixed operator. Nonetheless we can use the argument above to study the spectrum of lower order perturbations of $L$.

The spectrum of $L$ satisfies Weyl asymptotics, and therefore $(L+1)^{-p}$ is trace class, for large enough $p$. For $T$ we take a polyhomogeneous, pseudodifferential operator, satisfying the transmission condition with respect to $X$, of order $s<2$. For $a \geq 0$, such an operator satisfies estimates of the form:

$$
\|T f\|_{H^{a}(X)} \leq C_{s}\|f\|_{H^{s+a}(X)} \text { for } f \in \mathcal{C}^{\infty}(X) .
$$

The domain of the maximal operator defined by $T$ is therefore contained in $H^{s}(X)$, which contains the domain of $L$. As the operator $T(L+1)^{-1}$ is a bounded map from $L^{2}(X)$ to $H^{2-s}(X)$, it is compact from $L^{2}$ to $L^{2}$. As $(L+1)^{-1}$ belongs to a $p$-Schatten class, some power of $(L+1)^{-1} T(L+1)^{-1}$ is also trace class; Theorem 1 applies to show that $A=L+T$ is a closed operator with a compact resolvent. As $L$ is non-negative, all but finitely many eigenvalues lie in the right half plane. The argument used to prove (19) applies, mutatis mutandis, to show that there are constants $C_{1}, C_{2}$ such that

$$
\Lambda(A) \subset\left\{\sigma+i \tau:|\tau| \leq C_{1}\left(C_{2}+\sigma\right)^{\frac{t}{2}}\right\} .
$$


In this example, the domains of $A$ and $L$ are the same. It would be interesting to estimate the location of the spectrum for a boundary value problem, which is a "perturbation," in some sense, of a self adjoint, elliptic, or sub-elliptic, boundary value problem. As the operation of restriction to the boundary of $X$ is unbounded with respect to $L^{2}$, the problem of defining "small" perturbations of the boundary conditions is a rather subtle question, to which we will return in a subsequent publication.

\section{Acknowledgment}

I would like to thank the referee for his careful reading of, and useful remarks on my paper.

\section{REFERENCES}

[1] E. B. Davies, Non-self adjoint differential operators, Bull. London Math. Soc., 34 (2002), pp. 513-532.

[2] N. Dencker, J. Sjöstrand, And M. Zworski, Pseudospectra of semi-classical (pseudo)differential operators, Comm. Pure Appl. Math., 57 (2004), pp. 384-415.

[3] I. Gohberg and M. Krein, Introduction to the Theory of Linear Nonselfadjoint Operators, vol. 18 of Translations of Mathematical Monographs, A.M.S., Providence, 1969.

[4] T. Kato, Perturbation Theory for Linear Operators, corrected 2nd printing, vol. 132 of Grundlehren der mathematischen Wissenschaften, Springer Verlag, Berlin Heidelberg New York, 1980.

[5] L. N. Trefethen and M. Embree, Spectra and Pseudospectra: The Behavior of Nonnormal Matrices and Operators, Princeton University Press, Princeton, NJ, 2005.

[6] M. Zworski, A remark on a paper of E.B. Davies, Proc. of A.M.S., 129 (2001), pp. 29552957.

[7] _ Numerical linear algebra and solvability of partial differential equations, Comm. Math. Phys., 229 (2002), pp. 293-307.

\section{Charles L. Epstein}

Department of Mathematics, University of Pennsylvania,

209 S. 33rd Street, Philadelphia, PA 19104.

E-mail: cle@math.upenn.edu 